\title{
A new type of optical activity in a toroidal metamaterial
}

\author{
T. A. Raybould ${ }^{1}$, V. A. Fedotov ${ }^{1}$, N. Papasimakis ${ }^{1}$, I. Youngs ${ }^{2}$, W. T. Chen $^{3}$, D. P. Tsai ${ }^{3}$, and \\ N. I. Zheludev ${ }^{1,4}$ \\ ${ }^{1}$ Optoelectronics Research Centre and Centre for Photonic Metamaterials, University of Southampton, \\ SO171BJ, U.K. \\ ${ }^{2}$ DSTL, Salisbury, U.K. \\ ${ }^{3}$ Department of Physics, National Taiwan University, Taipei, 10617, Taiwan \\ ${ }^{4}$ Centre for Disruptive Photonic Technologies, Nanyang Technological University, Singapore
}

\begin{abstract}
Optical activity is a phenomenon which is widely observed across both natural and artificially chiral structures. Microscopically, the presence of optical activity in a system is conventionally understood in terms of the coupling of excited, co-aligned electric and magnetic dipoles[1]. Whilst combinations of electric dipoles with higher-order multipoles can also contribute to optical activity, such effects are frequently considered to be negligible. Metamaterial engineering however, allows for the design of structures where conventional microscopic multipole responses are suppressed in favour of more unusual excitations, including the elusive toroidal dipole.

First considered by Zel'dovich in 1958[2], the toroidal dipole has recently been identified as an integral component in the excitations of metamaterial structures[3]. As the toroidal dipole radiates as an electric multipole[4], it is anticipated that it could contribute to optical activity in the same manner as an electric dipole. Here, we report on the first observation of optical activity in a structurally-chiral metamaterial that cannot be attributed to conventional multipoles, and can only be accounted for by the inclusion of the toroidal dipole in the multipole expansion.

The metamaterial unit cell studied consists of four split-ring resonators embedded in a low-loss dielectric, with the unit cells arranged such that the material has a structural chirality. The metamaterial is studied experimentally in a microwave chamber and computationally using a commerical Maxwell's equation solver. By extracting the microscopic multipole response, we confirm that at resonance a non-negligible toroidal dipole is excited and contributes to the observed circular dichroism in the metamaterial's transmission spectrum. This is corroborated by an examination of the material's polarisation eigenstates and how they vary upon removal of multipole excitations from the metamaterial's response.
\end{abstract}

\section{ACKNOWLEDGMENT}

The authorsacknowledge support from DSTL, the Engineering and Physical Sciences Council, U.K. and the Leverhulme Trust.

\section{REFERENCES}

1. Barron, L. D., Molecular Light Scattering and Optical Activity, Cambridge Univ. Press, Cambridge, U.K., 1982.

2. Zel'dovich, I. B., "Electromagnetic interaction with parity violation," Sov. Phys. JETP, Vol. 6, 1184-1186, 1958.

3. Kaelberer, T. et al, "Toroidal dipolar response in a metamaterial," Science, Vol. 330, 1510$1512,1986$.

4. Dubovik, V. M. and Tugushev, V. V., "Toroid moments in electrodynamics and solid-state physics," Phys. Rep., Vol. 187, 145-202, 1990. 